\title{
A Radio-Frequency-over-Fiber link for large-array radio astronomy applications.
}

\author{
Juan Mena; Kevin Bandura, Jean-François Cliche, Matt Dobbs, Adam Gilbert, Qing \\ Yang Tang
}

Department of Physics, McGill University

3600 University Street, Montreal, Quebec H3A 2T8, Canada

E-mail: juan.menaparra@mail.mcgill.ca

\begin{abstract}
A prototype 425-850 MHz Radio-Frequency-over-Fiber (RFoF) link for the Canadian Hydrogen Intensity Mapping Experiment (CHIME) is presented. The design is based on a directly modulated Fabry-Perot (FP) laser, operating at ambient temperature, and a single-mode fiber. The dynamic performance, gain stability, and phase stability of the RFoF link are characterized. Tests on a two-element interferometer built at the Dominion Radio Astrophysical Observatory for CHIME prototyping demonstrate that RFoF can be successfully used as a cost-effective solution for analog signal transport on the CHIME telescope and other large-array radio astronomy applications.
\end{abstract}

KEYWORDS: Instrument optimisation; Interferometry; Lasers.

${ }^{*}$ Corresponding author. 


\section{Contents}

1. Introduction [1]

2. Analog signal chain and RFoF performance requirements 2]

3. RFoF link design and dynamic performance

4. Gain and phase stability of the RFoF link 9

5. RFoF Characterization on the CHIME two-element interferometer 13

6. Future work and conclusions

\section{Introduction}

The Canadian Hydrogen Intensity Mapping Experiment (CHIME) ${ }^{1}$ is currently under development at the Dominion Radio Astrophysical Observatory (DRAO) in Penticton, Canada. It is an array of cylindrical telescopes with more than 2500 radio receivers and has no moving parts or cryogenics. CHIME will map the three-dimensional distribution of neutral hydrogen gas in the universe by directly detecting its redshifted 21-cm radiation. By measuring the scale of the Baryon Acoustic Oscillations across the redshift range $\mathrm{z} \approx 0.8$ to $\mathrm{z} \approx 2.5$ in both the angular and line-of-sight directions, CHIME will study the epoch when Dark Energy generated the transition from decelerated to accelerated expansion of the universe.

As a prototype for testing the CHIME technology, a two-element radio interferometer was built at DRAO. While characterizing the CHIME analog receiver in the two-element interferometer, the implementation of a Radio-Frequency-over-Fiber (RFoF) link to transport the analog signals from the antennas to the processing room has been investigated as an alternative to traditional coaxial cable. Radio astronomy applications of RFoF are becoming common due, in part, to the pioneering implementation [1] for the Allen Telescope Array (ATA) []ㅡ. RFoF is an attractive option for the analog receiver in CHIME, and its advantages over coaxial cable include not only cost, but also small size and light weight, immunity to electromagnetic interference, low loss, and a separate grounding scheme for the front end radio frequency (RF) chain and the digital back end.

Analog transmission over a RFoF link also reduces the likelihood of self-generated radio frequency interference (RFI) pickup and reduces the amount of electronics and maintenance at the antennas compared to a receiver architecture with the digitizers located at the antennas and digital data transmission to the processing room. However, the analog optical link also introduces challenges that must be overcome, mainly related to linearity, noise, and stability. In this paper, a

1 www.CHIMExperiment.ca 


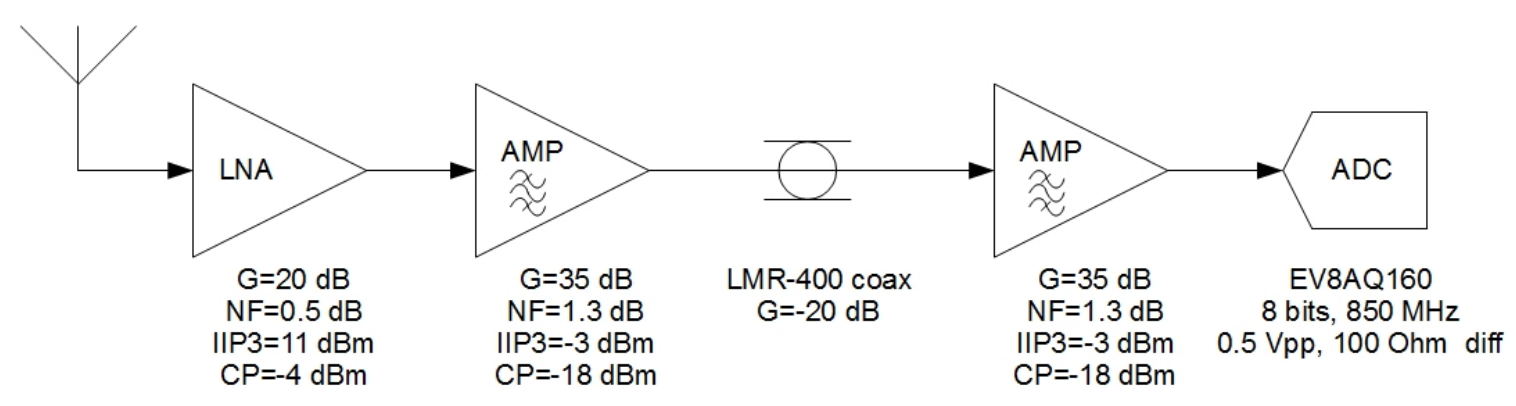

Figure 1. Coax-based receiver block diagram for one channel of the two-element interferometer. The gain (G), noise figure (NF), input third order intercept point (IIP3), and $1 \mathrm{~dB}$ input compression point (CP) shown for each block are referred to the block's input.

prototype version of a custom-built RFoF link for CHIME is presented, its dynamic performance, gain stability, and phase stability are characterized. The third order intercept point (IP3) is used as the figure of merit for the RFoF linearity and is appropriate for an octave-bandwidth system like CHIME. The corresponding spurious-free dynamic range (SFDR) is used as measure of the RFoF dynamic performance. We demonstrate a system wherein the noise and linearity are dominated by the 8-bit analog to digital converter (ADC) that follows the RFoF link, meaning that these challenges have been addressed at level that makes them negligible for the overall system.

This paper is structured as follows: Section 2 describes the present implementation of the ana$\log$ receiver for the two-element interferometer and the requirements on the dynamic performance of the RFoF link. Section 3 describes the analog optical link design and lab tests on its dynamic performance. In Section $\Psi$, test results on the gain and phase stability of the optical link with changes in temperature of the RFoF transmitter and the optical fiber are presented. The first tests of the RFoF link in the two-element interferometer are shown in Section 5. A final discussion on the overall RFoF performance and improvements for the next version of the link are presented in Section 6 .

\section{Analog signal chain and RFoF performance requirements}

A block diagram of the (coax-based) receiver for the CHIME two-element interferometer is shown in Figure 1. The interferometer consists of two $9 \mathrm{~m}$ diameter parabolic dish reflectors spaced $19 \mathrm{~m}$ apart on an east-west line. The feed for each dish is a four-square above a ground-plane and is based on the design presented in [3] . The amplifier chain consists of a custom low noise amplifier (LNA) followed by a second-stage amplifier and filter. The feed analog stages are in individually shielded boxes. A $50 \mathrm{~m}$ coaxial cable is connected to the focus receiver to transmit the signal to an electronics hut containing the digitizer and additional back end electronics. The $20 \mathrm{~dB}$ loss from the coax in Figure 1 includes the loss in both the cable and attenuators installed at each end of the cable. Inside the electronics hut, the signal passes through a second combined amplifier and filter box and is then fed into the digitizer and correlator. For a more detailed description of the CHIME two-element interferometer refer to [田]. 
The frequency response of the analog receiver is defined by a commercial band-pass filter with a $3 \mathrm{~dB}$ passband in the approximate range $475-800 \mathrm{MHz}$. The noise of the LNA is approximately $35 \mathrm{~K}(\mathrm{NF}=0.5 \mathrm{~dB})$ across the CHIME band and this is the main contribution to the noise of the receiver electronics. The output signals from the receiver are directly sampled at 850 MSPS, recovering the second Nyquist zone from $425-850 \mathrm{MHz}$. There is no analog down-conversion stage. The ADC samples are processed by a custom Field Programmable Gate Array (FPGA) based correlator using an FX (Fourier transform followed by cross-multiplication) architecture: The signals are first put through a poly-phase filter bank (PFB) and then Fourier transformed using a 2048-point Fast Fourier Transform. The data processing thereafter is separate for each frequency. The four signals (two dishes and two polarizations) are cross-multiplied and averaged to complete the correlation.

The RFoF link, having relatively high input noise $(\mathrm{NF} \approx 27 \mathrm{~dB})$, is the last stage of the receiver chain with its output connected directly to the ADC. A gain of at least $50 \mathrm{~dB}$ upstream of the RFoF input is required to make its noise contribution negligible in comparison to the front-end LNA. To ensure the RFoF link has negligible impact on the entire analog chain, it is required to have an output referred dynamic performance that is substantially better than that of the ADC. The SFDR of the RFoF is determined by its IP3 and noise floor. This requirement translates to an output IP3 (OIP3) much larger than the IIP3 of the ADC and an output noise floor much lower than the input noise of the $\mathrm{ADC}^{2}$.

For the RFoF link, the IP3 determines the level of the third-order intermodulation distortion products (IMD3) as a function of the output signal level. In contrast, an ADC does not have a single, well defined IP3. For a typical ADC, the IMD3 curve has three regions. For low level input signals, the IMD3 products remain relatively constant regardless of signal level. Near the ADC full-scale, the IMD3 products behave as expected ( $3 \mathrm{~dB}$ increase per every $\mathrm{dB}$ increase in the input power). The power level at which this begins depends on the specific ADC device. Above the full-range, the ADC acts as an ideal limiter and the IMD3 products become large due to clipping. For a detailed description of this subject see [5].

We determine the IP3 of the ADC by measuring the IMD3 products using two input tones such that the ADC is close to full range (which is typically the IMD3 value reported on ADC datasheets). A calculation of the ADC IP3 based on this measurement yields an optimistic value, and an overestimation of the ADC SFDR as long as the ADC is below full range ${ }^{3}$. Thus, requiring that the OIP3 of the RFoF is much greater than this measure of the ADC IIP3 is enough to guarantee that the distortion generated by the RFoF is much smaller than that generated by the ADC.

Both the two-element interferometer and the CHIME pathfinder (a prototype of CHIME consisting of two cylindrical telescopes with 256 total radio receivers that is currently under construction at DRAO) use the $\mathrm{E}^{2} \mathrm{~V}^{4}$ EV8AQ160 quad-channel, 8-bit ADC implemented on a custom

\footnotetext{
${ }^{2}$ In general, the board where the ADC is mounted has some attenuation, mainly due to input matching and singleended to differential input conversion for the ADC, so the input and output noise floor and IP3 of the ADC board will be different.

${ }^{3}$ In this document SFDR always refers to the spurious free dynamic range as calculated from the two-tone third order intermodulation distortion products. This should not be confused with the SFDR specified in ADC datasheets which is obtained from a single-tone test as the ratio of the signal power to the power of the strongest spurious signal.

4 http://www.e2v.com/
} 


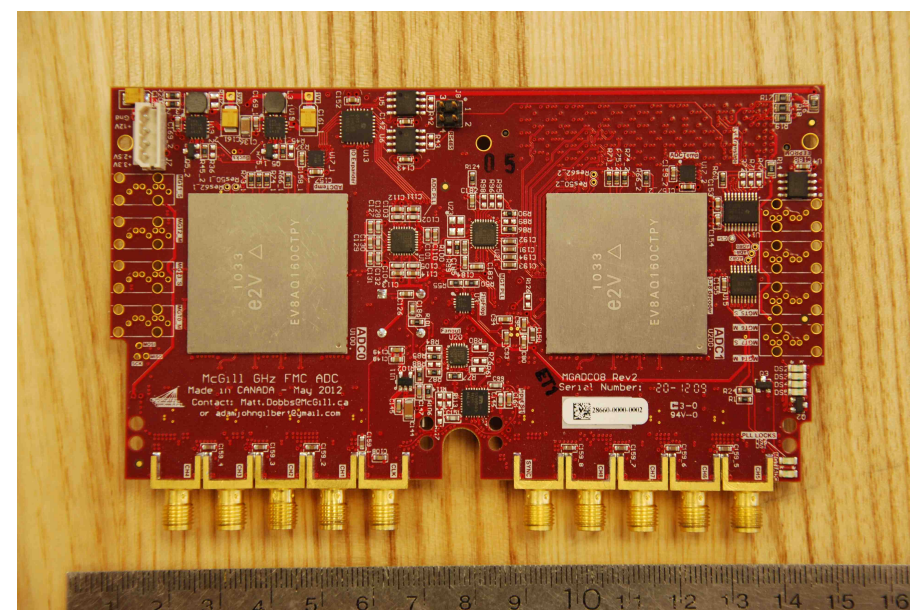

Figure 2. The custom-built ADC board for CHIME includes two ADCs that can each digitize four analog channels with 8 bits at a maximum sampling rate of 1.25 GSPS, for a total of eight channels per board. Each ADC channel streams its data across eight differential signals to the FPGA. For CHIME, the ADCs will be operated at 800 MSPS to perform alias sampling of the 400-800 MHz signal. The ADC board can interface to any FPGA motherboard that implements the full FMC High Pin Count standard. The two additional inputs are for the reference clock and the synchronization signal.

FPGA Mezzanine Card (FMC) compliant circuit board with two devices providing eight input channels. The insertion loss of the ADC board, from its single ended input to the differential ADC, is approximately $2 \mathrm{~dB}$. Each ADC device can sample at rates up to 1.25 GSPS in four-channel mode with a full range of $0.5 \mathrm{~V}_{\mathrm{p}-\mathrm{p}}$ differential input. The measurements presented in this paper are sampled at 850 MSPS whereas CHIME will operate at 800 MSPS. A top view of the custom ADC board is shown in Figure 2 .

The performance of the ADC is characterized by the SFDR. The IP3 of the ADC board was measured using two tones at $632.5 \mathrm{MHz}$ and $652 \mathrm{MHz}$. We obtained IIP3=13 dBm. The noise of the ADC board is determined from the signal-to-noise-and-distortion (SINAD) measurement of the ADC (typically parametrized as effective-number-of-bits, ENOB) and then combined with the IP3 measurement to obtain the SFDR. The measured ENOB is 6.9 bits at $632.5 \mathrm{MHz}$. The corresponding input noise floor is approximately $\mathrm{P}_{\mathrm{ni}}=-132 \mathrm{dBm} / \mathrm{Hz}$, resulting in SFDR $=97 \mathrm{~dB} \cdot \mathrm{Hz}^{2 / 3}$ for the ADC board.

\section{RFoF link design and dynamic performance}

The selection of the components and modulation method for the RFoF design is mainly driven by two parameters: cost and performance. Due to its simplicity, intensity modulation combined with direct detection (IMDD) is the most widely employed method to convey an RF signal over an analog optical link [ [ [ [] as compared to other modulation and detection techniques (e.g. frequency and phase modulation of the optical carrier combined with coherent detection). In IMDD the RF signal modulates the intensity of an optical carrier, which then travels over the optical fiber and is detected by a photodetector. Two ways to implement the IMDD method are to use external 
modulation or direct modulation. In an externally modulated system the laser operates in continuous wave mode and the modulation is done externally with an optical modulator. These are high performance systems that provide high dynamic range and large bandwidth over long transmission distances (e.g. the implementation for ATA [四], [园]). However, the need for the additional external modulator and high performance, high power lasers increases the system costs dramatically. This, in the case of a large-array application like CHIME requiring thousands of RFoF links, is a major disadvantage.

The custom-built RFoF link for CHIME is a directly modulated system. The main advantage of direct modulation is simplicity and low cost as compared to externally modulated systems. For a short-range application like CHIME, where the deterioration of the optical signal in the fiber is small, the performance of the link is mainly determined by the laser [G]. Distributed Feedback (DFB) lasers are generally preferred for analog applications due to their high linearity, high slope efficiency (the slope of the laser output power versus the laser current curve) and low noise characteristic compared to other lasers. However, these are the most expensive laser types due to their complicated fabrication [8]. Vertical-Cavity-Surface-Emitting Lasers (VCSELs) offer low cost and low power consumption due to the low threshold current. Their optical characteristics are similar to DFB lasers (a single wavelength peak is present in their spectrum) but their performance is, in general, below that of DFB lasers. Several VCSELs were tested in the lab as possible candidates for the RFoF prototype and SFDR values in the range $90-100 \mathrm{~dB} \cdot \mathrm{Hz}^{2 / 3}$ were achieved. A similar RFoF link using VCSELs for antenna remoting in the Australian Square Kilometre Array Pathfinder achieved $98 \mathrm{~dB} \cdot \mathrm{Hz}^{2 / 3}$ [9] [10]. These values are below the requirements for CHIME. The current RFoF prototype uses a Fabry-Perot (FP) laser, which for short-range applications offers good performance and its costs are lower than for DFB lasers. Due to its characteristic design, several wavelengths are present in the FP spectrum, which results in signal deterioration for transmission over distances exceeding a few kilometers due to dispersion in the fiber [6] [11] [12] [13] . For short-range applications like CHIME, this effect is very small and the constraints from the laser dominate. The performance of the link for distances larger than $100 \mathrm{~m}$ has not been investigated.

Figure 3 shows the prototype RFoF link design. The RF analog signal is placed on the optical carrier by directly modulating the laser current with the signal. The optical signal travels through the fiber and the photodiode converts the signal back to the electric domain for additional amplification and filtering. The RFoF transmitter features an $\mathrm{AGX}^{5}$ uncooled, linear multi-quantum well (MQW) FP laser emitting at $1310 \mathrm{~nm}$, hermetically sealed in an industry-standard coaxial package with a single-mode fiber pigtail and subscriber connector/angled physical contact (SC/APC) connector. Integrated in the laser package is an optical isolator to prevent unwanted feedback into the laser and a monitor photodiode to control the laser optical output power. Each RFoF transmitter is electrically sealed in an aluminum box.

The RFoF receiver uses an AGX linear, low-capacitance photodiode that is sensitive to the wavelength range between $1100 \mathrm{~nm}$ and $1650 \mathrm{~nm}$. This photodiode module is also a hermetically sealed coaxial package with a single-mode fiber pigtail and SC/APC connector. The prototype version of the RFoF receiver was constructed as a board with four receiver channels, all enclosed in the same aluminum box. Each RFoF receiver channel includes an amplification chain and the

5 http://www.agxtech.com/ 


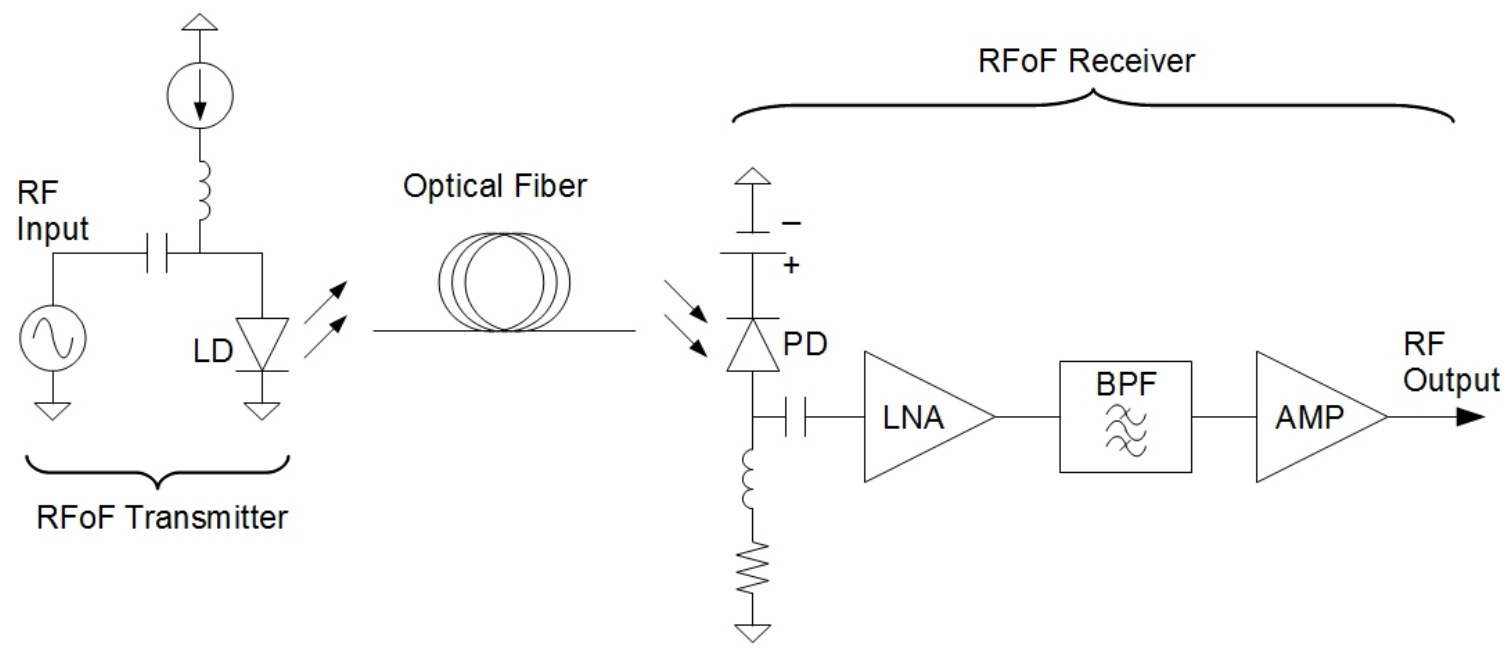

Figure 3. Schematic diagram of the prototype CHIME RFoF link. The laser (LD) current is directly modulated by the RF signal. The optical signal travels through the fiber and the photodiode (PD) converts the signal back to the electric domain. The RFoF receiver includes an amplification chain and the same bandpass filter (BPF) as the second stage amplifier of the two-element interferometer receiver.

same band defining filter as the second stage amplifier of the two-element interferometer receiver in Figure 1. A top view of the single-channel RFoF transmitter and four-channel receiver is shown in Figure 4 . The approximate cost is US\$ 200 per link in quantities of several hundred, not including the cost of fiber. The laser and the band-pass filter dominate the cost.

The performance of the RFoF was characterized with a suite of laboratory tests. All the measurements in this section are performed at $25{ }^{\circ} \mathrm{C}$ using a $80 \mathrm{~m}$ fiber. The optical output power feedback control circuitry of the RFoF transmitter has been disabled for these tests as explained in Section $\theta$. Without the RFoF receiver amplification chain, the gain of the link is approximately -20 $\mathrm{dB}$, a value which is mainly determined by the slope efficiency of the laser and the responsivity of the photodiode [6] [14]. Amplification is needed in the receiver to boost the OIP3 of the RFoF to a level well above that of the ADC, while keeping the output noise floor below the input noise of the ADC. This is implemented in two stages. A commercial LNA is placed after the photodiode, followed by the band defining filter and a commercial high IP3 amplifier for the final amplification stage. An amplification greater than the electric-optical-electric conversion loss is required to have margin which allows for attenuation pads at each stage, improving the impedance matching and reducing the in-band ripple from the filter. The end-to-end gain of the RFoF link in the CHIME band has been set to $4 \mathrm{~dB}$ with a corresponding OIP3 of approximately $29 \mathrm{dBm}$. Measurements of the scattering parameters for the RFoF link are shown in Figure 5 and measurements of the output noise, CP, OIP3 and crosstalk between adjacent RFoF receiver channels are shown in Figure 6. A summary of the characteristics and dynamic performance of the RFoF link in the CHIME band is shown in Table [1.

The main contribution to the noise of the RFoF link is the Relative Intensity Noise (RIN) of the laser. Since the dispersion effects introduced by the fiber are small near $1310 \mathrm{~nm}$ and for the transmission distances considered in CHIME (approximately $100 \mathrm{~m}$ ), the noise penalty introduced 


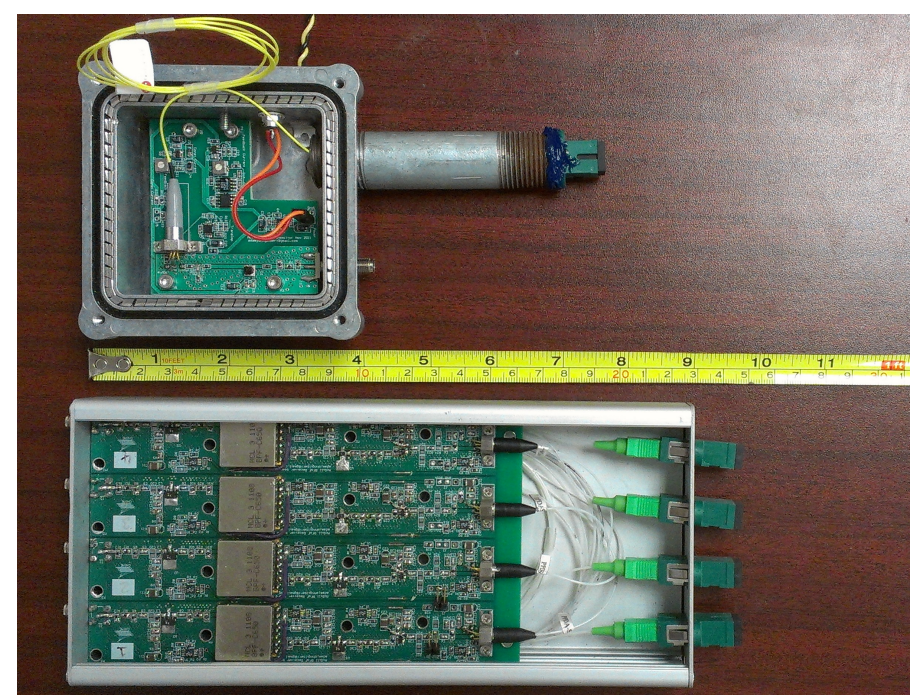

Figure 4. The RFoF transmitter (top) features an uncooled, linear MQW FP laser emitting at $1310 \mathrm{~nm}$, hermetically sealed in an industry-standard coaxial package with a single-mode fiber pigtail and SC/APC connector. Each RFoF transmitter is electrically sealed in a dedicated aluminum box. The RFoF receiver (bottom) uses a linear, low-capacitance photodiode sensitive to the wavelength range between $1100 \mathrm{~nm}$ and $1650 \mathrm{~nm}$. This photodiode module is also a hermetically sealed coaxial package with a single-mode fiber pigtail and SC/APC connector. The first version of the RFoF receiver was constructed as a board with four receiver channels, all enclosed in the same aluminum box. Each RFoF receiver channel includes an amplification chain and the same band defining filter as the second stage amplifier of the two-element interferometer receiver.

Table 1. Characteristics and dynamic performance of the RFoF link in the $475-800 \mathrm{MHz}$ band. All measurements are performed at $25^{\circ} \mathrm{C}$ using an $80 \mathrm{~m}$ fiber.

\begin{tabular}{|c|c|c|}
\hline Parameter & Symbol & Value \\
\hline Operating wavelength & $\lambda$ & $1310 \mathrm{~nm}$ \\
\hline Laser operating bias current & $\mathrm{I}_{\text {bias }}$ & $30 \mathrm{~mA}$ \\
\hline Laser optical output power & $\mathrm{P}_{\mathrm{opt}}$ & $3.0 \mathrm{~mW}$ \\
\hline $1 \mathrm{~dB}$ (input) compression point & $\mathrm{CP}$ & $>10 \mathrm{dBm}$ \\
\hline Gain & $\mathrm{G}$ & $4 \mathrm{~dB}$ \\
\hline Input return loss & $\left|\mathrm{S}_{11 \mathrm{~dB}}\right|$ & $>16 \mathrm{~dB}$ \\
\hline Output third order intercept & $\mathrm{OIP} 3$ & $29 \mathrm{dBm}$ \\
\hline Output noise floor & $\mathrm{P}_{\mathrm{no}}$ & $-143 \mathrm{dBm} / \mathrm{Hz}$ \\
\hline Noise figure & $\mathrm{NF}$ & $27 \mathrm{~dB}^{2 / 3}$ \\
\hline Spurious-free dynamic range & $\mathrm{SFDR}$ & $115 \mathrm{~dB} \cdot \mathrm{Hz}^{2 / 3}$ \\
\hline
\end{tabular}

by the spectral impurity of the FP laser (e.g. Mode Partition Noise, a form of noise that arises from the random distribution of the photons produced by stimulated emission in each wavelength of the laser spectrum) is negligible [13] [15]. The RFoF link has $\mathrm{NF}=27 \mathrm{~dB}\left(\mathrm{P}_{\mathrm{ni}}=-147 \mathrm{dBm} / \mathrm{Hz}\right)$ and 


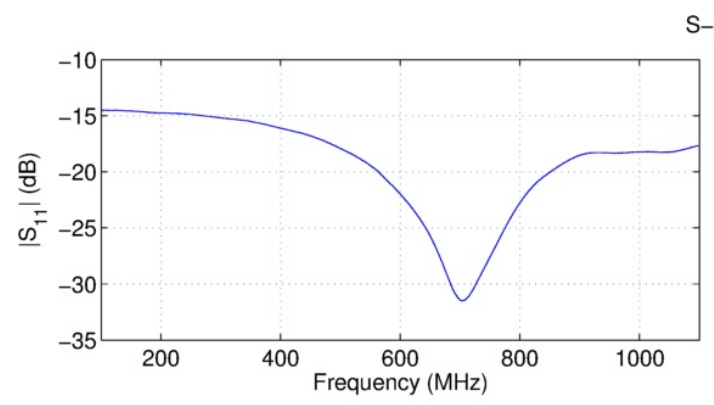

S-parameters for RFoF
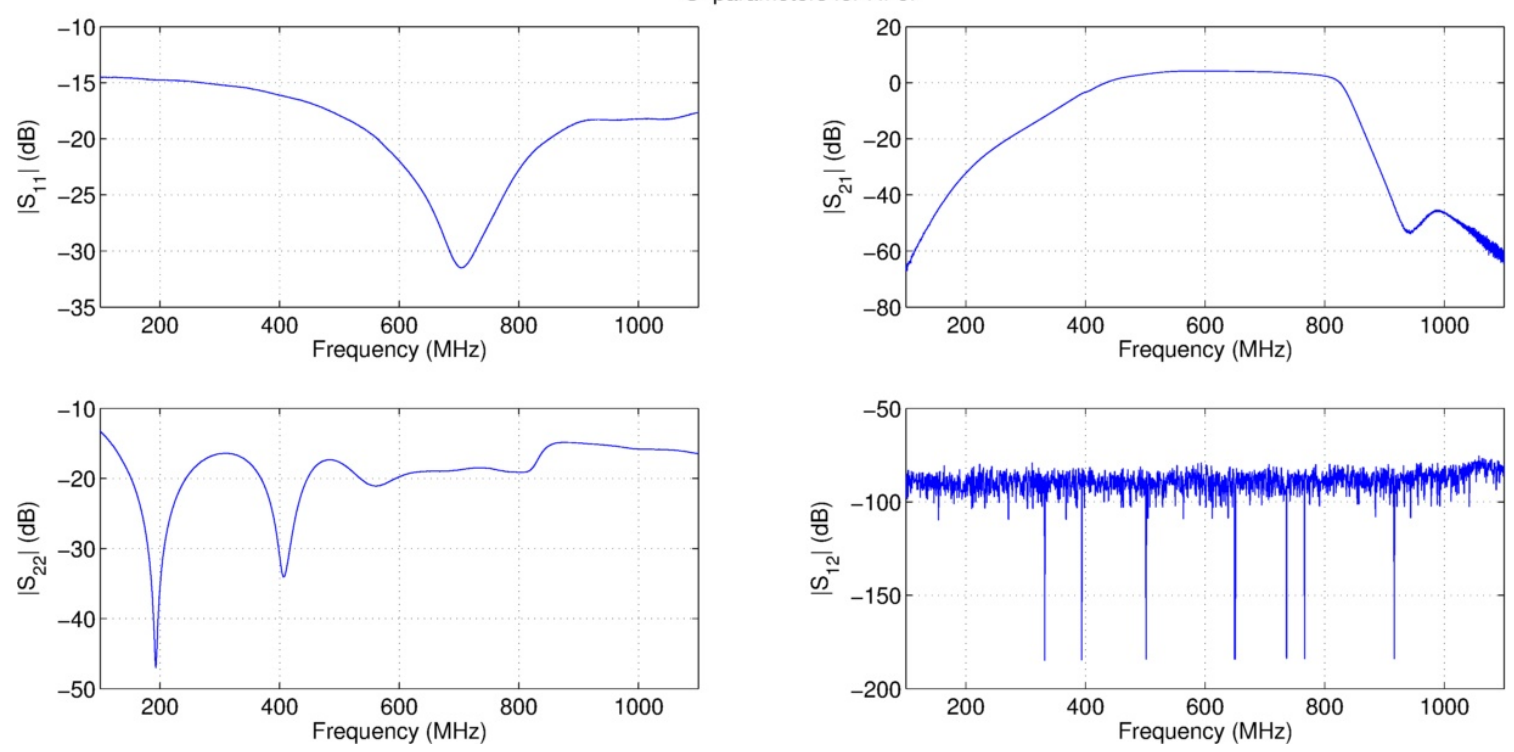

Figure 5. Scattering parameters of the RFoF link. An $80 \mathrm{~m}$ fiber was used. $S_{11}$ (top left) and $S_{22}$ (bottom left) are, respectively, the input and output reflection coefficients of the link. $S_{21}$ (top right) and $S_{12}$ (bottom right) are, respectively, the forward and reverse gains. The average gain in the $475-800 \mathrm{MHz}$ band is approximately $4 \mathrm{~dB}$. All measurements are performed at $25{ }^{\circ} \mathrm{C}$. The $\mathrm{S}_{12}$ measurement is limited by the dynamic range of the Network Analyzer used.

the corresponding SFDR is $115 \mathrm{~dB} \cdot \mathrm{Hz}^{2 / 3}$, or $57 \mathrm{~dB}$ in a $425 \mathrm{MHz}$ bandwidth. These results are well matched to an 8-bit ADC, as they will have a negligible impact on the noise and linearity of the receiver chain. This performance over $80 \mathrm{~m}$ distances is comparable to that of more expensive high performance DFB laser-based RFoF links for radio astronomy applications (e.g. [16], [17], [18]).

The degradation of the ADC's SFDR introduced by the RFoF link can be estimated by calculating the noise and IP3 of the cascaded RFoF+ADC system at the input of the RFoF using the method of [19]. The input noise of the cascaded system is

$$
\mathrm{P}_{\mathrm{ni}}=10 \log \left(10^{\mathrm{P}_{\text {no RFoF }} / 10}+10^{\mathrm{P}_{\mathrm{ni}} \mathrm{ADC} / 10}\right)-\mathrm{G}_{\mathrm{RFoF}} \approx-135.7 \mathrm{dBm} / \mathrm{Hz} .
$$

where $\mathrm{P}_{\text {no RFoF }}$ and $\mathrm{P}_{\mathrm{ni}} \mathrm{ADC}$ are the output noise of the RFoF link and input noise of the ADC board (in $\mathrm{dBm} / \mathrm{Hz}$ ), respectively, and $\mathrm{G}_{\mathrm{RFoF}}$ is the gain of the RFoF link (in $\mathrm{dB}$ ). The IIP3 of the cascaded system is

$$
\mathrm{IIP} 3=-10 \log \left(10^{-\mathrm{OIP} 3_{\mathrm{RFoF}} / 10}+10^{-\mathrm{IIP} 3_{\mathrm{ADC}} / 10}\right)-\mathrm{G}_{\mathrm{RFoF}} \approx 8.9 \mathrm{dBm} .
$$

where OIP $3_{\mathrm{RFoF}}$ and IIP $3_{\mathrm{ADC}}$ are the OIP3 of the RFoF link and IIP3 of the ADC board (in $\mathrm{dBm}$ ), respectively. Thus, the SFDR of the cascaded system is $\mathrm{SFDR} \approx 96.4 \mathrm{~dB} \cdot \mathrm{Hz}^{2 / 3}$, a reduction of less than $1 \mathrm{~dB}$ with respect to the SFDR of the ADC alone.

The crosstalk performance between adjacent RFoF receiver channels is better than $45 \mathrm{~dB}$ in the CHIME band. The crosstalk requirement for CHIME is set by the ADC board, which has a 

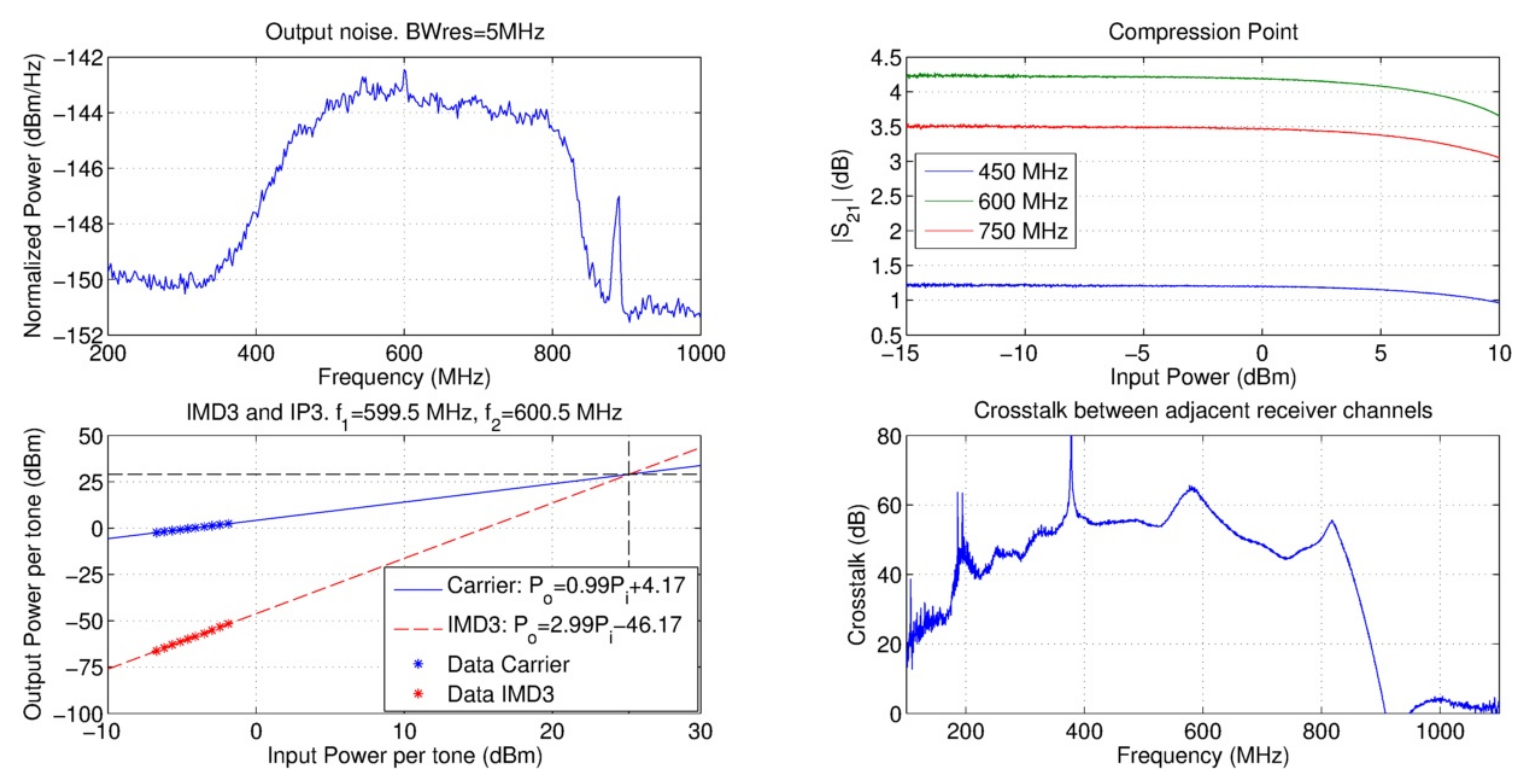

Figure 6. Output noise (top left), Compression Point (top right), IP3 (bottom left) and crosstalk between adjacent receiver channels (bottom right) of the RFoF link at $25{ }^{\circ} \mathrm{C}$ using an $80 \mathrm{~m}$ fiber. The average normalized output noise power in the $475-800 \mathrm{MHz}$ band (top left) is $\mathrm{P}_{\mathrm{no}}=-143 \mathrm{dBm} / \mathrm{Hz}$ and the out-ofband noise floor is limited by the dynamic range of the Spectrum Analyzer. The CP exceeds $10 \mathrm{dBm}$ and the measurement (top right) is limited by the maximum output power of the Network Analyzer. The OIP3 is approximately $29 \mathrm{dBm}$ (bottom left) measured using two tones at $599.5 \mathrm{MHz}$ and $600.5 \mathrm{MHz}$. The crosstalk performance between adjacent $\mathrm{RFoF}$ receiver channels (bottom right) is better than $45 \mathrm{~dB}$ across the CHIME band.

minimum crosstalk performance better $55 \mathrm{~dB}$ between adjacent channels in the CHIME band. In order to meet this requirement, the production version of the RFoF link for CHIME will use an aluminum shielded box for each individual RFoF receiver channel.

\section{Gain and phase stability of the RFoF link}

The gain and phase stability of the analog receiver are critical for radio interferometry. Gain and phase fluctuations, resulting mainly from temperature effects in the components of the receiver, generate errors in the visibility data and loss in sensitivity [20]. The receiver stability determines how often the calibration procedure, such as the injection of a known signal across the array, needs to be performed.

In the case of the CHIME two-element interferometer, the RFoF receiver is located in a temperature controlled electronics hut with the back end electronics. Thus it is expected that the gain and phase stability of the RFoF will be driven by temperature fluctuations of the transmitter and the fiber, which are in an outdoor environment. Measurements as a function of temperature will be presented separately for these two components below.

Measurements of the gain and phase variation of the RFoF link with changes in temperature of the RFoF transmitter box are shown in Figure 7. The RFoF transmitter was placed inside a climate controlled chamber and the temperature was varied from $-25^{\circ} \mathrm{C}$ to $45^{\circ} \mathrm{C}$ in steps of 

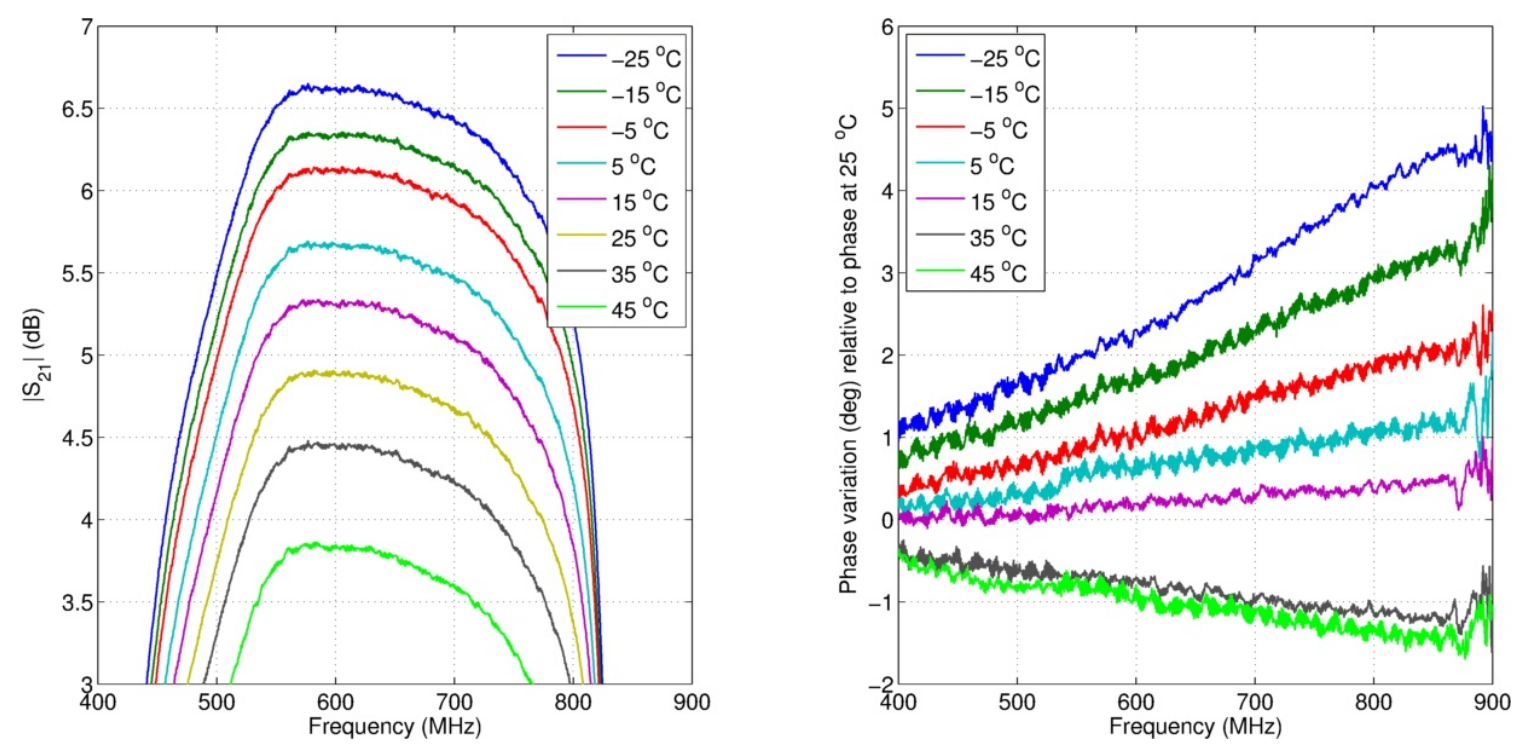

Figure 7. Gain (left) and phase (right) variation of the RFoF link with changes in temperature of the RFoF transmitter. An $80 \mathrm{~m}$ fiber was used. The temperature of the RFoF receiver and fiber was kept at $25^{\circ} \mathrm{C}$. The phase variation is shown as the phase difference with respect to the phase at $25^{\circ} \mathrm{C}$.

$10{ }^{\circ} \mathrm{C}$. This encompasses the expected range of temperatures at DRAO. Both the RFoF receiver and the $80 \mathrm{~m}$ fiber were kept at $25^{\circ} \mathrm{C}$ during the measurements. The phase variation is shown as $\arg \left(\mathrm{S}_{21}(T)\right)-\arg \left(\mathrm{S}_{21}\left(T=25^{\circ} \mathrm{C}\right)\right)$, where $\arg \left(\mathrm{S}_{21}\left(T=25^{\circ} \mathrm{C}\right)\right)$ is the phase of $\mathrm{S}_{21}$ at $25^{\circ} \mathrm{C}$ and $\arg \left(\mathrm{S}_{21}(T)\right)$ is the phase of $\mathrm{S}_{21}$ at the temperature of the chamber. The in-band gain variation is approximately $3 \mathrm{~dB}$ for a $70{ }^{\circ} \mathrm{C}$ temperature change, or approximately $0.04 \mathrm{~dB} /{ }^{\circ} \mathrm{C}$, mainly caused by the change in the slope efficiency of the FP laser. The phase variation is below $6^{\circ}$ in-band for a $70{ }^{\circ} \mathrm{C}$ temperature change.

The RFoF transmitter includes an automatic optical power control module for the laser. When the module is enabled, higher gain variations were observed. This is due to a combination of a decrease in the slope efficiency of the laser as the temperature increases and the fact that the $\mathrm{P}_{\text {opt }}$ vs. $\mathrm{I}_{\text {bias }}$ curve of the laser starts to compress as the current is increased. Since the main purpose of the optical power control system is to keep the laser bias current well above the threshold current of the laser (which increases with temperature), it is possible to disable the module as long as the laser bias current, which is now fixed, is well above the threshold current along the full range of expected temperatures for the RFoF transmitter. The power control module has been disabled for the measurements presented in this paper and it will be removed for the production version of the RFoF link for CHIME.

In addition, the nonlinearity and noise performance of the RFoF link as a function of the temperature of the RFoF transmitter were measured. Within the uncertainty of the measurements (about $1 \mathrm{~dB}$ ), the OIP3, output noise, and consequently the SFDR remained constant during the test (at $29 \mathrm{dBm},-143 \mathrm{dBm} / \mathrm{Hz}$, and $115 \mathrm{~dB} \cdot \mathrm{Hz}^{2 / 3}$ respectively). However, the IIP3 and NF rose with temperature: the IIP3 changed from $23 \mathrm{dBm}$ at $-25^{\circ} \mathrm{C}$ to $26 \mathrm{dBm}$ at $45^{\circ} \mathrm{C}$ while the $\mathrm{NF}$ changed from $25 \mathrm{~dB}$ at $-25^{\circ} \mathrm{C}$ to $28 \mathrm{~dB}$ at $45^{\circ} \mathrm{C}$. 

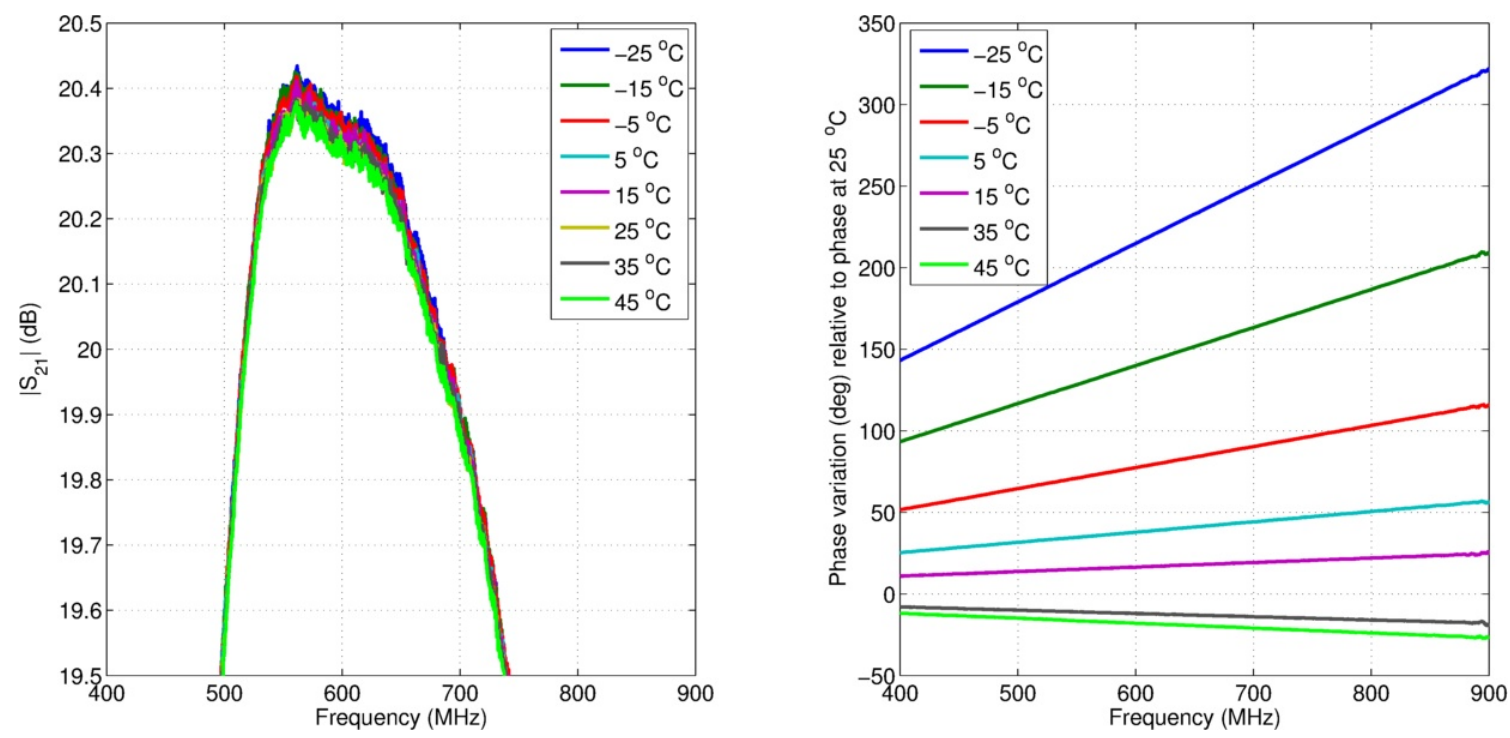

Figure 8. Gain (left) and phase (right) variation of the RFoF link with changes in temperature of the fiber. An $80 \mathrm{~m}$ fiber was used. The temperature of the $\mathrm{RFoF}$ transmitter and receiver was kept at $25^{\circ} \mathrm{C}$. The phase variation is shown as the phase difference in degrees with respect to the phase at $25^{\circ} \mathrm{C}$.

Measurements of the gain and phase variation of the RFoF link with changes in temperature of the fiber are shown in Figure 8. The $80 \mathrm{~m}$ fiber was placed inside the chamber and the temperature was varied from $-25^{\circ} \mathrm{C}$ to $45^{\circ} \mathrm{C}$ in steps of $10^{\circ} \mathrm{C}$. A standard $9 / 125 \mu \mathrm{m}$ tight buffer patch cord was used. The gain variation is less than $0.1 \mathrm{~dB}$ for a $70{ }^{\circ} \mathrm{C}$ temperature change, very small compared to the gain variation due to the RFoF transmitter. However, the phase variation of the fiber is relatively high and will be the dominant source of phase variations for the interferometer. It is common to express the phase stability of the fiber in terms of the Thermal Coefficient of Delay (TCD) defined as

$$
\mathrm{TCD}=\frac{\mathrm{d} \tau}{\mathrm{d} T} \frac{10^{6}}{\tau} \approx \frac{\Delta \phi}{\phi} \frac{10^{6}}{\Delta T}
$$

where $\tau$ is the insertion delay and $\phi$ is the respective insertion phase of the fiber. $\mathrm{d} \tau / \mathrm{d} T$ is determined by variations in the length and refractive index of the fiber [21]. Figure 9 shows the estimated TCD obtained from the phase information in Figure 8 assuming that the fiber has a velocity factor of 0.68 and a length of $80 \mathrm{~m}$ under standard conditions $\left(25^{\circ} \mathrm{C}\right)$. The TCD is approximately $90 \mathrm{ppm} /{ }^{\circ} \mathrm{C}$ at $-25^{\circ} \mathrm{C}$, relatively high compared to LMR-400 coax cable, for which cable providers typically specify a TCD below $10 \mathrm{ppm} /{ }^{\circ} \mathrm{C}$. However, the phase stability of the optical link can be greatly improved by using loose tube fiber, in which the manufacturing process ensures that the fiber is protected from stresses caused by temperature changes and mechanical forces [22]. For this cable design the TCD reduces to values below $10 \mathrm{ppm} /{ }^{\circ} \mathrm{C}$ [21] and, in general, has a better performance in outdoor applications. Since the change to loose tube causes no increase in the cost of the cable, this will be the fiber used for the production version of the CHIME RFoF link.

A method commonly used for the reduction of gain fluctuations in the optical link is to control the temperature of the laser by means of a thermistor and a thermoelectric cooler, with both 


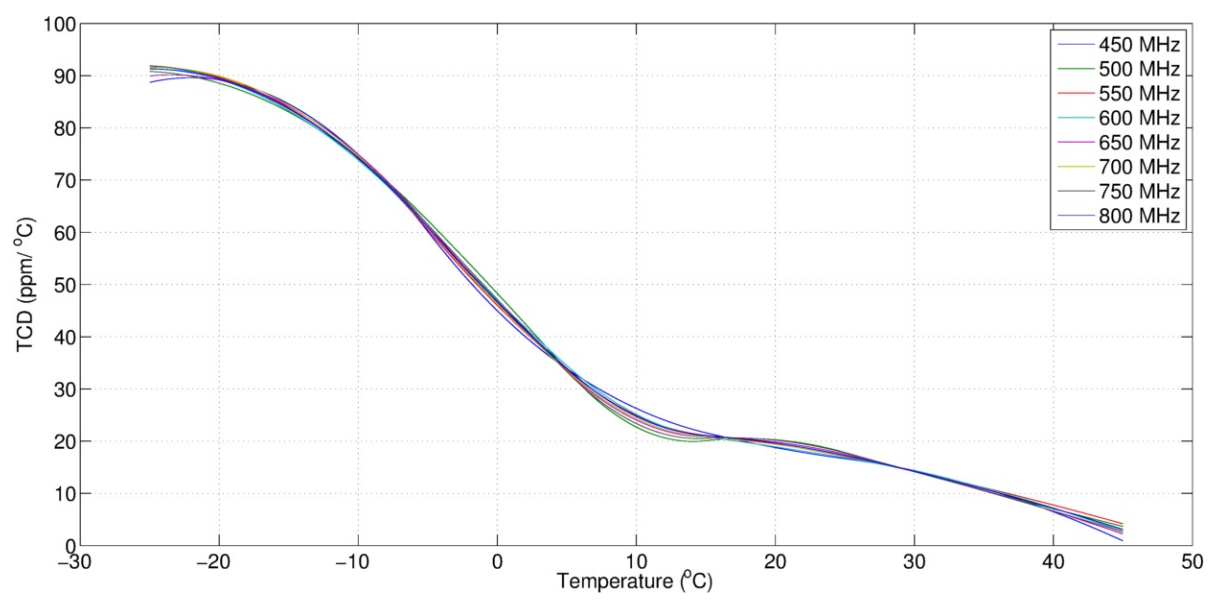

Figure 9. Estimated TCD of the fiber as a function of temperature. A velocity factor of 0.68 and a length of $80 \mathrm{~m}$ under standard conditions $\left(25^{\circ} \mathrm{C}\right)$ was assumed for the fiber. Note that $\phi$ cannot be obtained directly from $\mathrm{S}_{21}$ since the insertion phase of the other components of the RFoF link has not been measured.

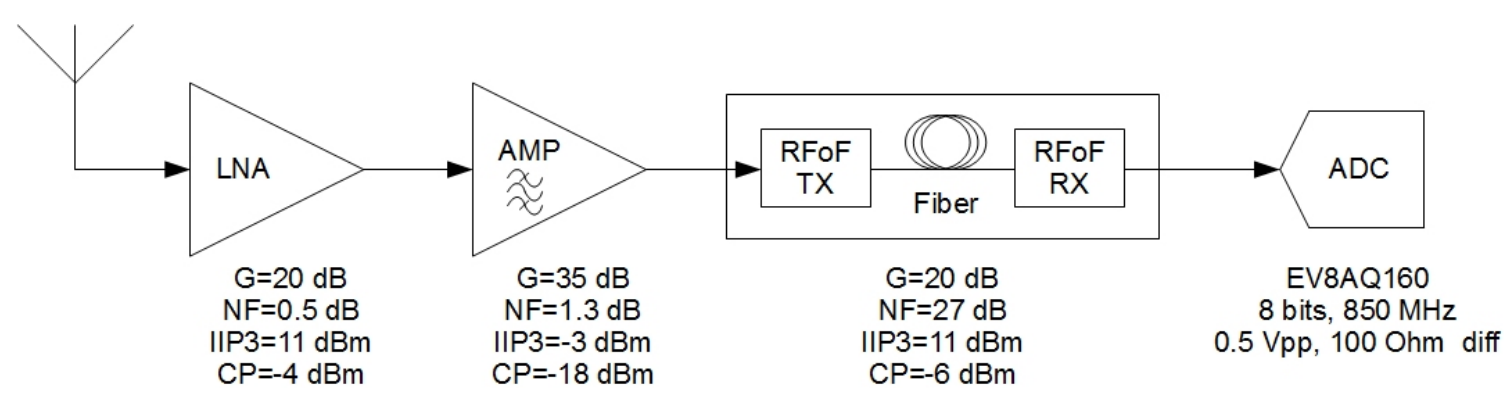

Figure 10. Two-element interferometer receiver block diagram using RFoF. The parameters of each block are referred to its respective input.

components either in a separate module on the RFoF transmitter board or included in the laser package. The latter option is discarded for CHIME because cooled lasers are typically an order of magnitude more expensive than uncooled ones. Instead of trying to reduce the gain fluctuations by analog means, a much simpler and more cost-effective alternative is to perform a gain compensation of the analog receiver implemented in firmware or software after the digitization or during the calibration procedure. This is feasible as long as the signal degradation introduced by ADC quantization and noise is acceptable across the full range of temperature induced gain variations of the analog receiver.

In order to characterize this effect, the gain variation of the analog receiver with RFoF was also investigated. A diagram of the receiver chain with RFoF is shown in Figure 10. The current analog receiver design requires a $20 \mathrm{~dB}$ gain $\mathrm{RFoF}$ link in order to achieve the desired noise power at the input of the ADC. Optimally, the additional gain must be added at the input of the RFoF link in order to keep the RFoF output noise level below that of the ADC. For this test, the additional gain was added in the RFoF receiver so we could use the existing analog receiver electronics. The effect of this change in the overall system performance is negligible since the noise performance of the 

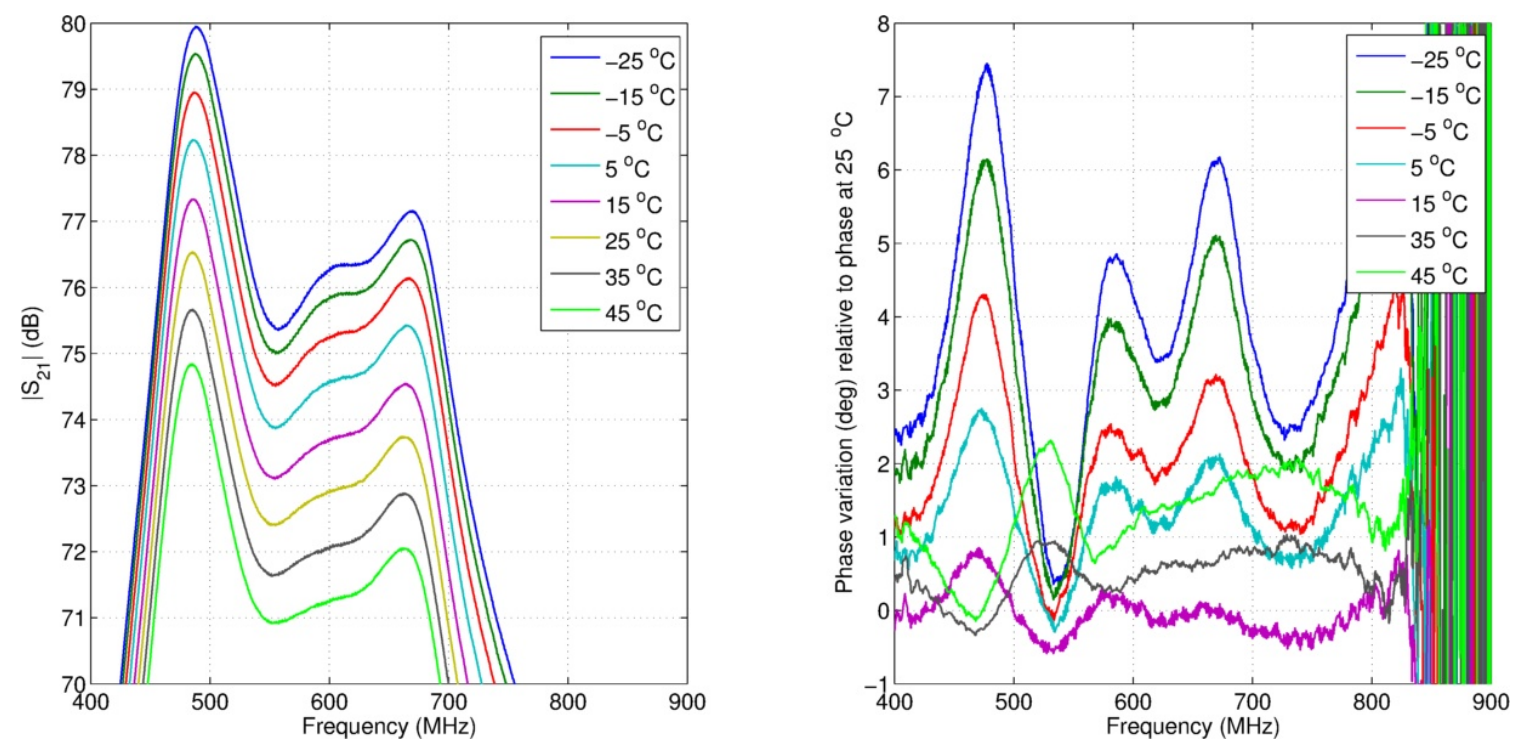

Figure 11. Gain (left) and phase (right) variation of the RFoF-based two-element interferometer receiver with changes in temperature of the elements at the focus. An $80 \mathrm{~m}$ fiber was used. The temperature of the $\mathrm{RFoF}$ receiver and fiber was kept at $25^{\circ} \mathrm{C}$. The phase variation is shown as the phase difference with respect to the phase at $25^{\circ} \mathrm{C}$.

system is still dominated by the LNA and its nonlinear behavior is still dominated by the ADC. The production version of the RFoF link will have the additional gain in the transmitter. For this test, all the components of the receiver which are located at the focus (LNA, second-stage amplifier and $\mathrm{RFoF}$ transmitter) were placed in the chamber and the temperature inside was varied from $-25^{\circ} \mathrm{C}$ to $45^{\circ} \mathrm{C}$. The results are shown in Figure [1]. The in-band gain variation is approximately $5 \mathrm{~dB}$ for a $70{ }^{\circ} \mathrm{C}$ temperature change, or approximately $0.07 \mathrm{~dB} /{ }^{\circ} \mathrm{C}$.

The signal degradation due to quantization and 8-bit ADC noise is shown in Figure 12. For a detailed discussion of the fractional increase in the noise of a Gaussian distributed input signal that results from quantization refer to [20]. For the two-element interferometer, the typical input power to each channel of the analog receiver is estimated as $100 \mathrm{~K}$ in a $425 \mathrm{MHz}$ bandwidth, or about $-92 \mathrm{dBm}$ [ 四]. The average gain of the receiver in the $425-850 \mathrm{MHz}$ band is approximately $72 \mathrm{~dB}$ at $25^{\circ} \mathrm{C}$ which, including the $2 \mathrm{~dB}$ insertion loss of the ADC board, results in $-22 \mathrm{dBm}$ power at the input to the ADC or a voltage standard deviation of about 3.7 bits. This level causes a negligible noise penalty due to quantization and ADC noise, while allowing headroom for receiver linearity and ADC saturation caused by external RFI. The total variation in the gain of the receiver (from $-25^{\circ} \mathrm{C}$ to $45^{\circ} \mathrm{C}$ ) results in an input signal variation between 3.4 bits (at $45^{\circ} \mathrm{C}$ ) and 4.2 bits (at $-25^{\circ} \mathrm{C}$ ). As Figure 12 shows, the minimum of the quantization efficiency function is broad and the noise penalty at these levels remains negligible, so the gain calibration can be performed after digitization.

\section{RFoF Characterization on the CHIME two-element interferometer}

The first tests of the RFoF link on the CHIME two-element interferometer at DRAO were per- 


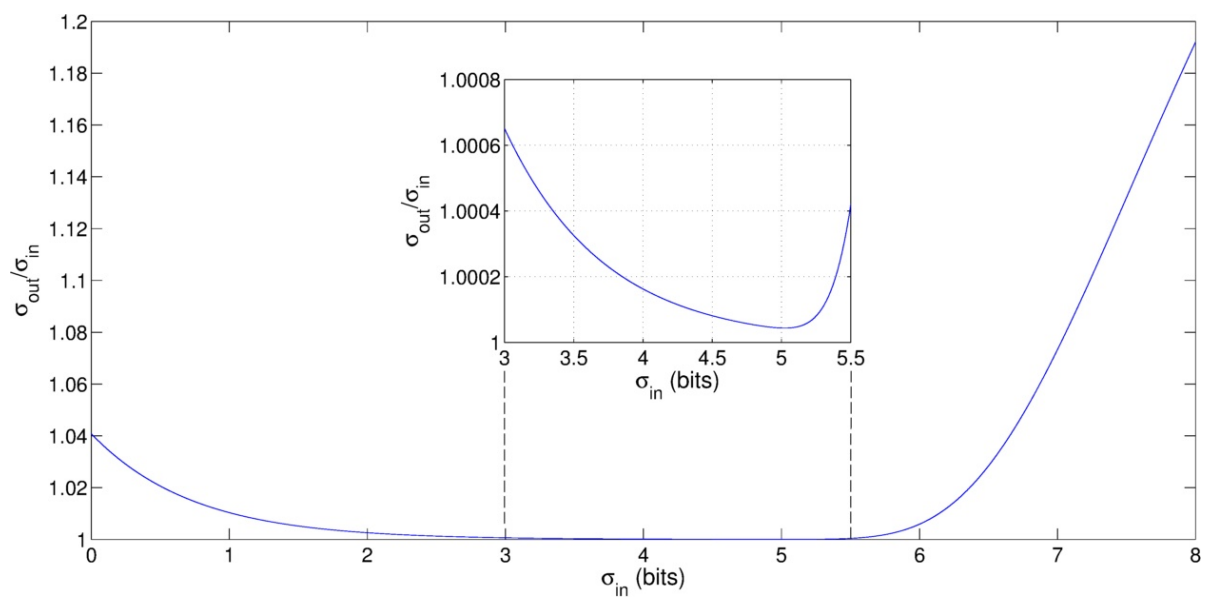

Figure 12. Fractional increase in the noise of a signal that results from the quantization with an 8-bit ADC. The input to the ADC is assumed to be gaussian noise with standard deviation $\sigma_{\mathrm{in}}$. The standard deviation of the signal after quantization is $\sigma_{\text {out }}$.

formed in January 2013. The purpose of these tests was to compare the performance of the RFoFbased receiver to that of the coax-based receiver. At the focus of each dish, the output of the feed is sent through the LNA, second-stage amplifier, and filter box. It is then split with one output connected to the ADC through the RFoF system (Figure 10) while the other output connected to the ADC through coax cable and a second combined amplifier and filter box located inside the hut (Figure 1).

With this setup, we proceeded to compare the noise performance of the two systems. The system temperature was calculated from 24 hours of raw data recorded in drift-scan mode and comparing it to the $408 \mathrm{MHz}$ Haslam map [23], excluding the bright radio source Cas A. The Haslam map was convolved with a Gaussian kernel determined by the beam parameters of the dish, and scaled using an estimated spectral index $\alpha_{e s t}=-2.5$ (see [四] for details). As Figure [13 shows, the noise performance of the coax-based and fiber-based receiver are consistent to within about $5 \mathrm{~K}$ across the band, which falls within the systematic uncertainty of this measurement. The system temperature of both systems is roughly $T_{s y s} \approx 100 \mathrm{~K}$ midband, of which about $40 \mathrm{~K}$ is caused by ground spill and loss in the feed. The spectral ripple in the $T_{\text {sys }}$ measurement is caused by standing waves between the ground plane and the reflector.

\section{Future work and conclusions}

In this paper, a prototype version of a low cost RFoF link for CHIME has been presented. The RFoF was characterized with a suite of laboratory tests and a very good dynamic performance was achieved, comparable to more expensive high performance DFB laser-based RFoF links used elsewhere for radio astronomy applications. In addition, the gain and phase stability of the RFoF link were investigated. It was shown that the gain fluctuations for the fiber-based CHIME receiver cause only a small noise penalty due to quantization and ADC noise of an 8-bit ADC, so the gain calibration can be performed after digitization. Finally, it was shown that there is no measurable 

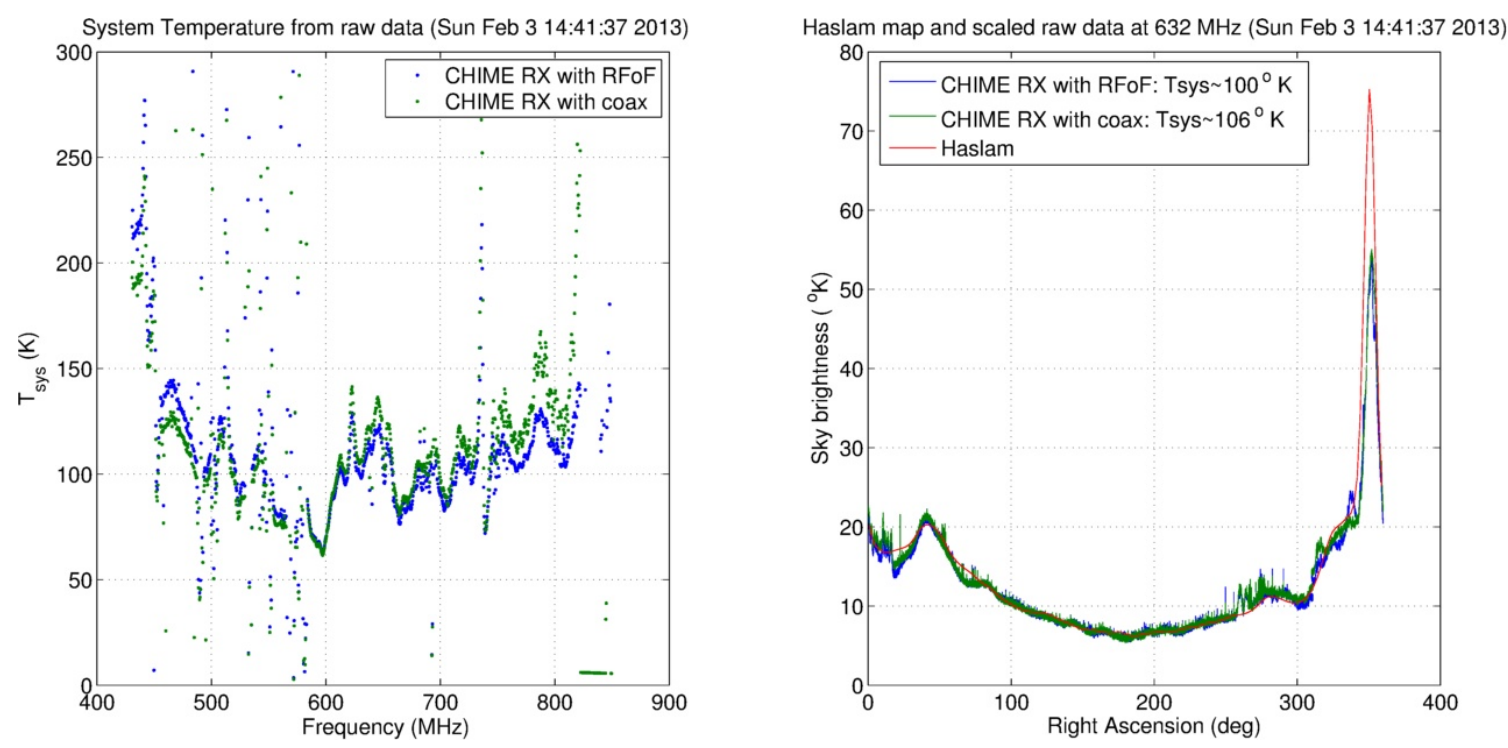

Figure 13. Estimated system temperature, $T_{s y s}$, for the coax-based and fiber-based receiver across the CHIME band from raw data measured by comparing to the Haslam map excluding Cas A (left). The Haslam map was convolved with a Gaussian kernel determined by the beam parameters of the dish, and scaled using an estimated spectral index $\alpha_{e s t}=-2.5$. A particular fit at $632 \mathrm{MHz}$ is shown in the right plot.

noise degradation as compared to the coax-based receiver. Near term improvements to this RFoF system will include separating the RFoF receivers into individually shielded boxes to improve the crosstalk performance and using loose tube fiber to improve the phase stability in an outdoor environment. Valuable experience was obtained during the design and characterization of the RFoF link for CHIME, demonstrating that RFoF can be successfully applied for analog signal transport in large-array radio astronomy applications at low cost.

\section{Acknowledgments}

We thank the DRAO staff and members of the CHIME collaboration for their comments and stimulating discussions. We acknowledge funding from the Natural Sciences and Engineering Research Council of Canada, the Canadian Institute for Advanced Research, and the Canadian Foundation for Innovation. MD acknowledges support from the Canada Research Chairs program. JM acknowledges support from the Fonds de recherche du Québec-Nature et technologies.

\section{References}

[1] Ackerman E., Cox III C., Dreher J., Davis M., DeBoer D., Fiber-optic antenna remoting for radio astronomy applications, 27th General Assembly of International Union of Radio Science (URSI), Maastricht, the Netherlands, pp. 1-4, 2004.

[2] Welch J. et al., The Allen Telescope Array: The first widefield, panchromatic, snapshot radio camera for radio astronomy and SETI, Proceedings of the IEEE, vol.97, No.8, pp.1438-1447, 2009, [arXiv:0904.0762v1 [astro-ph.IM]]. 
[3] Leung M., A wideband feed for a cylindrical radio telescope, PhD Thesis, University of Sydney, 2008.

[4] Davis G., A two element interferometer prototype for the Canadian Hydrogen Intensity Mapping Experiment, MSc Thesis, University of British Columbia, 2009.

[5] Kester W., Data conversion handbook, Elsevier/Newnes, Amsterdam 2005.

[6] Cox III C., Analog optical links: theory and practice, Cambridge University Press, New York 2004.

[7] Iezekiel S., Microwave photonics: devices and applications, John Wiley \& Sons, Chichester (United Kingdom) 2009.

[8] Ramaswami R., Sivarajan K., Sasaki G., Optical networks: a practical perspective, Morgan Kaufmann, Amsterdam 2009.

[9] Beresford R., ASKAP photonic requirements, International meeting on microwave photonics, jointly held with the 2008 asia-pacific microwave photonics conference, pp. 62-65, 2008.

[10] DeBoer D., et al., Australian SKA pathfinder: A high-dynamic range wide-field of view survey telescope, Proceedings of the IEEE, Vol. 97, No. 8, pp. 1507-1521, 2009.

[11] Capmany J., Martínez A., Ortega B., Pastor D., Transfer function of analog fiber-optic systems driven by Fabry-Perot lasers, Journal of the Optical Society of America B, Vol. 22, No. 10, pp. 2099-2106, 2005 .

[12] Lau K., Ultra-high frequency linear fiber optic systems, $2^{\text {nd }}$ Edition, Springer, Heidelberg (Germany) 2011.

[13] Wentworth R., Bodeep G., Darcie T., Laser Mode Partition Noise in lightwave systems using dispersive optical fiber, Journal of Lightwave Technology, Vol. 10, No. 1, pp. 84-89, 1992

[14] Cox III C., Ackerman E., Helkey R., Betts G., Techniques and performance of intensity-modulation direct-detection analog optical links, IEEE Transactions on Microwave Theory and Techniques, Vol. 45, No. 8, pp. 1375-1383, 1997.

[15] Suhara T., Semiconductor laser fundamentals, CRC Press, New York 2004.

[16] Montebugnoli S., Bianchi G., Monari J., Naldi G., Perini F., Schiaffino M., BEST: Basic Element for SKA Training, Proceedings of Wide Field Astronomy \& Technology for the Square Kilometre Array (SKADS), 2009.

[17] Montebugnoli S., Boschi M., Perini F., Faccin P., Brunori G., Pirazzini E., Large antenna array remoting using radio-over-fiber techniques for radio astronomical application, Microwave and Optical Technology Letters, Vol. 46, No. 1, pp. 48-54, 2005.

[18] Perini F., Analogue optical links experiences in the framework of the SKA/BEST activities, Proceedings of Wide Field Astronomy \& Technology for the Square Kilometre Array (SKADS), 2009.

[19] Bahl I., Fundamentals of RF and microwave transistor amplifiers, John Wiley \& Sons, Hoboken (New Jersey) 2009.

[20] Thompson A., Moran J., Swenson G., Interferometry and synthesis in radio astronomy, Wiley-VCH, $2^{\text {nd }}$ Edition, New York 2001.

[21] Bergman L., Johnston A., Temperature stability of transit time delay for a single-mode fibre in a loose tube cable, Electronics Letters, Vol.19, No.21, pp. 865-866, 1983 .

[22] Pal B., Fundamentals of fibre optics in telecommunication and sensor systems, Wiley, New York 1992.

[23] Haslam C., Salter C., Stoffel H., Wilson E., A 408 MHz all-sky continuum survey. II - The atlas of contour maps, Astronomy and Astrophysics Supplement Series, Vol. 47, pp. 1, 2, 4-51, 53-142, 1982. 